\title{
Resistive current states in narrow superconducting films
}

\author{
D A DIKIN, V M DMITRIEV, V N SVETLOV and \\ G E CHURILOV \\ Institute for Low Temperature Physics and Engineering, UkrSSR Academy of Sciences, \\ Kharkov, USSR
}

\begin{abstract}
The I-V characteristics of superconducting channels are studied experimentally in the region of low $\left(j \sim j_{c}\right)$ and high $\left(j \gg j_{c}\right)$ transport currents. The analytical expressions obtained for the I- $\mathrm{V}$ characteristics by Ivlev and Kopnin provide a good description of the experiment. The theoretical results suggest the existence of two temperature regions where the relaxation of the charge disbalance proceeds in different ways.
\end{abstract}

Keywords. Superconductivity; resistive state; critical current: excess current.

\section{Introduction}

The resistive current states and the effects of non-stationary and non-equilibrium superconductivity present one of the most important problems in the physics of superconductors. The development of the resistive state theory (Bezugly et al 1977) yielded analytical expressions for I-V characteristics (IVC) of channels in the vicinity of the critical temperature $T_{c}$ (Ivlev et al 1980; Ivlev and Kopnin 1984). It is therefore possible to compare experimental and theoretical IVCs of such objects, which is the subject of this paper.

\section{Experimental procedure}

The experiment was conducted on superconducting tin films $w \leqslant \xi(T), \lambda(T)$ wide and $d \ll \xi(T)$ thick. The parameters of two typical samples are given in table 1 .

A quantitative comparison of experimental and calculated IVCs (Ivlev et al 1980) requires the knowledge of electron-phonon relaxation time $\tau_{\varepsilon}$ for the sample in question, since this magnitude enters, as a parameter, into the expression describing IVCs and is responsible for the temperature range within which the appropriate formulae are valid. The measurement of the differential resistance $R_{g}$ from the linear part of IVC corresponding to the first phase-slip centre (PSC) can give the penetration depth of the longitudinal electric field $l_{E}(T)$ permitting subsequent calculation of $\tau_{\varepsilon}$ (Skocpol et al 1974). The obtained value $\tau_{\varepsilon}=1.5 \times 10^{-10} \mathrm{~s}$ is used below.

\section{Experimental study of IVC}

The typical IVCs taken on the whole sample Sn 1 (curve 3) and its two parts (curves 1,2) are given in figure 1 . The broken straight lines show IVC of the sample at normal state at $4.2 \mathrm{~K}$. The current and voltage scale fails to display the jump-like structure of IVCs in the low current region. According to Ivlev et al (1980), the sample Sn 1 is a long channel since $L \gg l_{E}(T)$. Hence, the IVC should look like a smooth curve, which can be 
Table 1. The parameters of the samples.

\begin{tabular}{|c|c|c|c|c|c|c|}
\hline \multirow[b]{2}{*}{ Nos. } & \multirow{2}{*}{$\begin{array}{c}\text { Sample } \\
\text { length, L } \\
10^{-6} \mathrm{~m}\end{array}$} & \multirow{2}{*}{$\begin{array}{l}\text { Width, w } \\
10^{-6} \mathrm{~m}\end{array}$} & \multirow[b]{2}{*}{$\begin{array}{l}\text { Thickness, } \\
\text { d, } 10^{-6} \mathrm{~m}\end{array}$} & \multicolumn{2}{|c|}{$\begin{array}{l}\text { Resistance at } \\
\text { temperature }\end{array}$} & \multirow[b]{2}{*}{$T_{c},(\mathrm{~K})$} \\
\hline & & & & $\begin{array}{l}T=300 \mathrm{~K} \\
R_{300}, \mathrm{Ohm}\end{array}$ & $\begin{array}{l}T=4.2 \mathrm{~K} \\
R_{4.2}, \mathrm{Ohm}\end{array}$ & \\
\hline Sn 1 & 101 & 1.5 & 0.1 & 88 & 7 & 3.847 \\
\hline Sn 2 & 40 & $2 \cdot 0$ & 0.35 & $7 \cdot 55$ & $0 \cdot 126$ & $3 \cdot 733$ \\
\hline
\end{tabular}

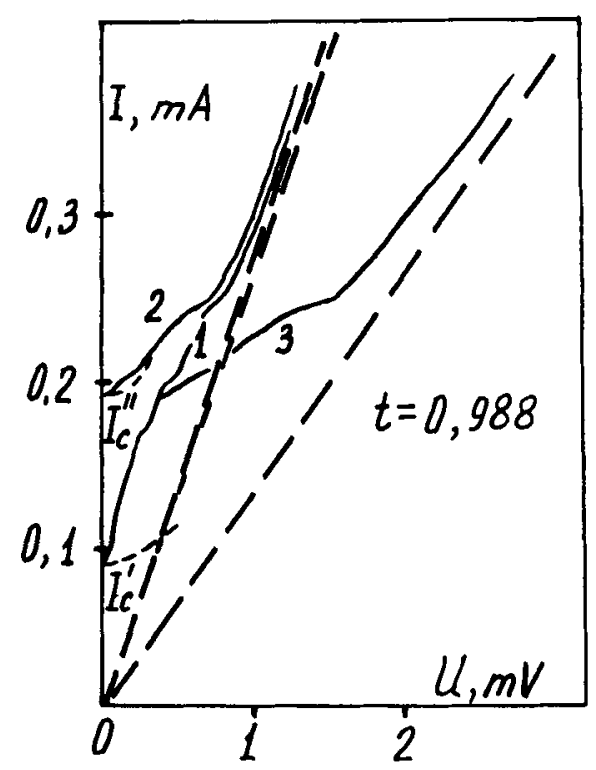

Figure 1. Experimental IVCs for Sn 1 (curve 3) and its two parts (curves 1,2). Two broken curves from $I_{c}^{\prime}$ and $I_{c}^{\prime \prime}$ are the IVC calculated by equation (1).

described by the following expressions (Ivlev et al 1980):

$$
J=J+J_{c} \exp \left(-0,91 J_{c} R_{4.2} / V\right)
$$

in the temperature interval

$$
\left(\tau_{\varepsilon} k T_{c} / \hbar\right)^{-2} \ll 1-t \ll\left(\tau_{\varepsilon} k T_{c} / \hbar\right)^{-1}
$$

and

$$
J=J_{c}+J_{c} \exp \left\{-1,1 \frac{J_{c} R_{4,2}}{V} \ln ^{\frac{1}{2}}\left[\tau_{\varepsilon} k\left(T_{c}-T\right) / \hbar\right]\right\}
$$

in the temperature interval

$$
\left(\tau_{\varepsilon} k T_{c} / \hbar\right)^{-1} \ll 1-t \ll\left(\tau_{\varepsilon} k T_{c} / \hbar\right)^{-\frac{1}{2}} / \ln \left[\tau_{\varepsilon} k\left(T_{c}-T\right) / \hbar\right] .
$$

The temperature intervals (2) and (4) are responsible for the effect of the superconducting current upon the relaxation rate of the phase-slip-induced disbalance between the 


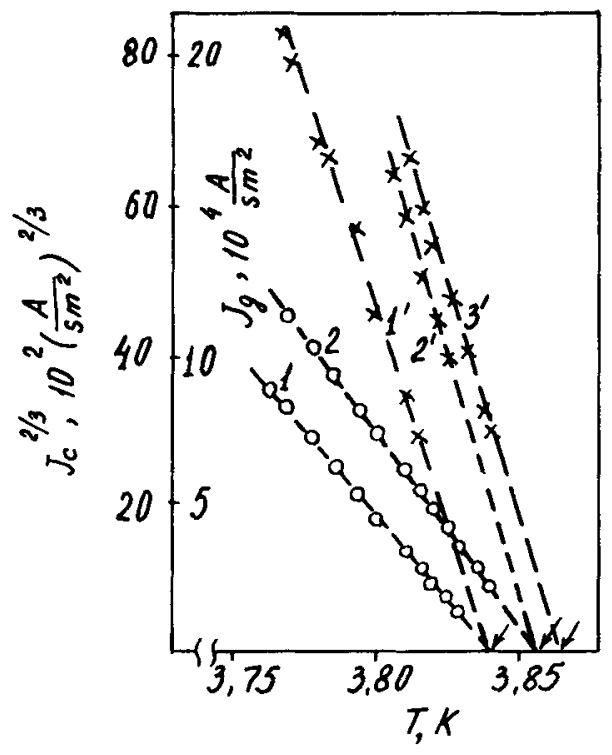

Figure 2. Temperature dependence of critical current density $J_{c}$ for two parts of the sample (curves 1,2 ) and non-Josephson generation current density $J_{q}$ (curves $1^{\prime}, 2^{\prime}, 3^{\prime}$ ) for Sn 1 .

chemical potentials of the pairs and quasi-particles (Ivlev et al 1980). At the temperatures corresponding to (2) this effect on the above disbalance is negligible, while at the temperatures (4) it becomes significant. For sample Sn 1 the temperature intervals (2) and (4) correspond to $0.986 \ll t \ll 0.9998$ and $0.93 \ll t \ll 0.986$, respectively. Because of high overheatings at low temperatures and fluctuations near $T_{c}$, the experimental range is $0.98-0.995$.

In addition to the experimental IVC of the whole sample (curve 3), figure 1 also shows the one calculated by equation (1) (a broken curve). No agreement is seen between the theoretical and experimental curves. This may result from the fact that the uniformity condition of the order parameter $\Delta$ (or $T_{c}$ ) is not obeyed along the sample length. If $R(T)$ is taken separately on two parts of $\mathrm{Sn} 1$, we have two values of $T_{c}$ differing by $\simeq 15 \times 10^{-3} \mathrm{~K}$. This $T_{c}$ variation for two parts of the sample is obtained when $T_{c}$ is calculated from the temperature dependence of the critical current (figure 2, straight lines 1,2). Moreover, finding $T_{c}$ from the temperature dependence of the non-Josephson generation current density $j_{g}$ (Churilov et al 1986) gives the third value of $T_{c}$ (figure 2 , straight lines $\left.1^{\prime}, 2^{\prime}, 3^{\prime}\right)$. The $T_{c}$ variation along the sample would lead to the situation when the critical currents of individual parts, which are at the same absolute but varying reduced temperatures can be several times different since $j_{c} \sim(1-t)^{3 / 2}$. In our case $j_{c}^{\prime}$ of one part of the sample differs nearly by a factor of two from $j_{c}^{\prime \prime}$ of the other part. The geometric nonuniformities at the edge or along the thickness, which do not exceed $10 \%$, produce much smaller effect on local $j_{c}$ values.

It is easier to meet the requirement of $T_{c}$ uniformity in shorter samples, which is seen in figure 1, where experimental curve 2 is closer to the calculated (broken) curve running from the point $I_{c}^{\prime \prime}$ than curve 3 from the point $I_{c}^{\prime}$.

The IVCs of the sample $\operatorname{Sn} 2$, whose total length is $L \simeq 4 \mathrm{l}_{E}(T)$, are given in figure 3 . The dispersion of $T_{c}$ data along the sample length is not more than $1 \times 10^{-3} \mathrm{~K}$; 


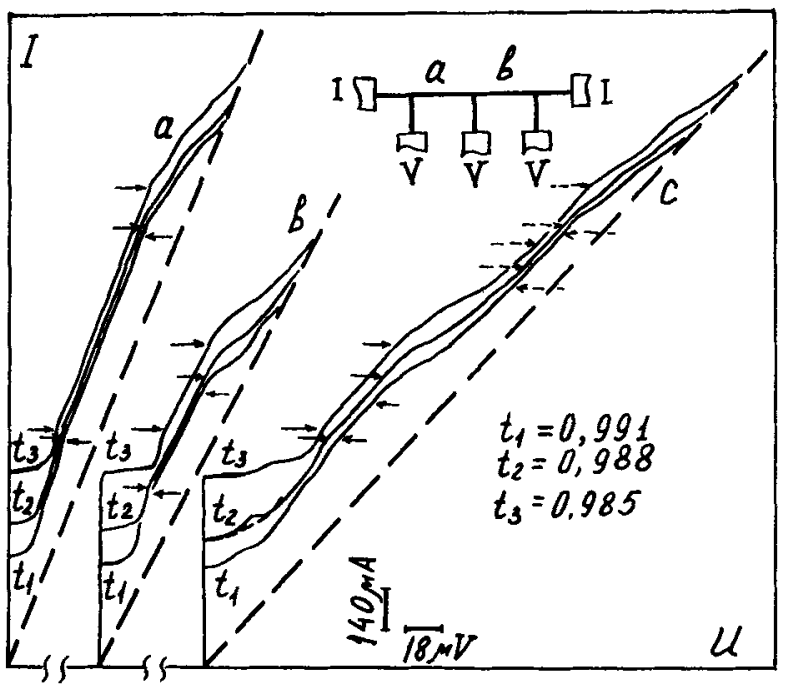

Figure 3. IVCs for Sn 2 taken on part $a$, part $b$, on the whole sample $c$ at various reduced temperatures $t$. Arrows show IVC portions with excess current. The broken curve for $t_{2}(c)$ is the initial portion of IVC calculated by equation (1).

accordingly, the critical currents of the two parts are practically the same. The dependence calculated by equation (1) for the initial part of IVC (figure $3 \mathrm{c}$, the broken curve for $t_{2}$ ) describes well the experimental one.

\section{Excess current in narrow superconducting films}

At $j-j_{c} \gg j_{c}$ the experimental IVC taken on a superconducting film reaches the linear part with $R_{g}=R_{4.2}$. This part of the IVC corresponds to the sample state with an excess current $j_{\text {exc }}$ and is described as follows:

$$
j=V / R_{4.2}+j_{\mathrm{exc}} \text {. }
$$

According to Ivlev et al (1980) in the temperature interval (2)

$$
j_{\mathrm{exc}}=a j_{c},
$$

where $a \simeq 0.68$, while in the temperature interval (4)

$$
J_{\text {exc }}=J_{c} b / \ln ^{\frac{1}{2}}\left[\tau_{\varepsilon} k\left(T_{c}-T\right) / \hbar\right],
$$

where $b \simeq 1.22$.

The relatively uniform sample $\mathrm{Sn} 2$ is schematically shown in figure 3 (insert). Seen are three groups of IVCs. Here $a$ and $b$ are the IVCs of the two corresponding parts of the sample, $c$ is the IVC of the whole sample.

As seen in figure 3, after the PSC formation, each part containing one centre, a linear part appears in the IVC, which is parallel to the straight line referring to the normal state. The resistive region is however shorter for part $b$ than for part $a$. Thus, the transition to the pure normal state in part $b$ occurs earlier than in part $a$. This may be associated, for example, with different conditions of the 


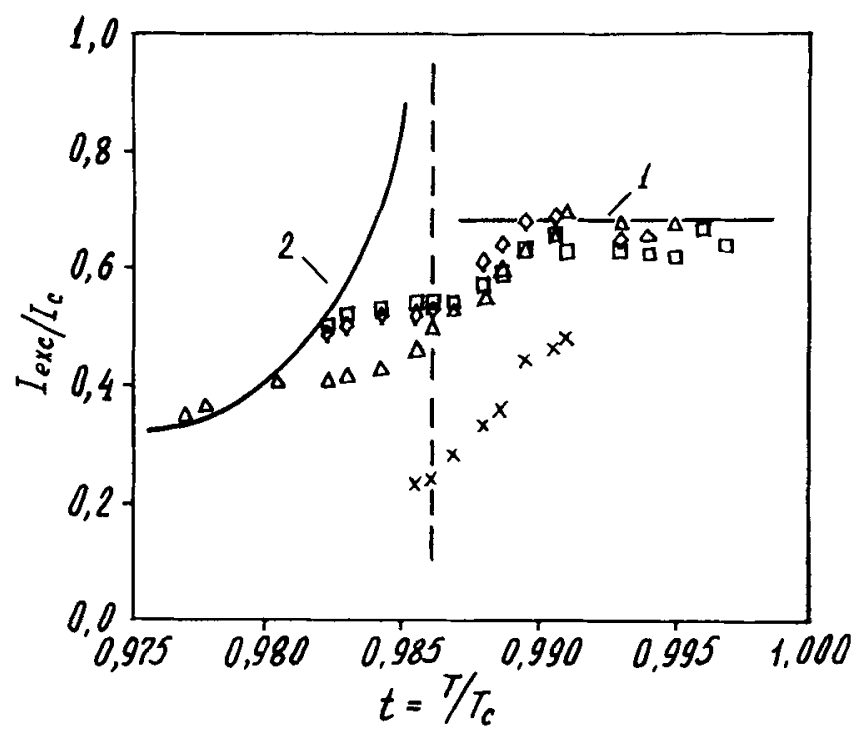

Figure 4. Dependence of excess current normalized to the critical current upon $t$ for Sn 2 . Experimental results were taken on part $a(\square)$, part $b(\Delta)$, the whole sample $(\diamond)$ (see figure $3 c)$, on the whole sample in portions marked with arrows in figure $3 \mathrm{c}(x)$. Solid straight line 1 is theoretical dependence (6), curve 2 is theoretical dependence (7) with numerical factor 0.2 .

thermal balance along the sample length because of varying degree of adhesion between the film and the substrate. The arrows on the total IVC show two parts of the IVC, which can be considered as the states with excess current. The part outlined with solid arrows indeed refers to the state with excess current of the whole sample. The region outlined with broken curves refers to the resistive state with excess current of part $a$, while part $b$ is already normal.

Figure 4 shows the temperature dependence $j_{\text {exc }} / j_{c}$ for separate parts of the film $(\square, \triangle)$ and for the whole sample $(\diamond)$ in conformity with figure 3 . The series of experimental points $(x)$ in figure 4 reflect, as we have found out, the situation, when one part of the film is normal and the other is still resistive. It is this situation that leads to about a two-fold decrease in $j_{\text {exc }} / j_{c}$, since both $a$ and $b$ parts have close $R_{4.2}$ values. Solid straight line 1 in figure 4 shows the theoretical dependence (6) and curve 2 the dependence (7) multiplied by 0.2 . As seen in the figure, the experimental values of $j_{\text {exc }} / j_{c}$ agree fairly well with the theoretical ones within the temperature interval (2), where the current effect on the disbalance relaxation rate is negligible. At lower temperature there is a transient region, in which the behaviour of the $j_{\text {exc }} / j_{c}$ magnitude has not been determined analytically. At still lower temperature the experimental points fall on to the theoretical dependence (7), the fitting factor 0.2 being taken into account.

In non-uniform samples of the Sn 1 type $j_{\text {exc }}$ is usually $(0.1-0.2) j_{c}$.

\section{Conclusion}

Thus, it is shown for the first time that the analytical expressions derived in Ivlev et al (1980) for IVCs in the regions of low $\left(j \sim j_{c}\right)$ and high $\left(j \gg j_{c}\right)$ currents describe well the experimental IVC for superconducting channels. Experimental support is provided for 
the theoretical results suggesting the existence of two temperature regions in which the relaxation of the charge disbalance proceeds in different ways: in one region, near $T_{c}$, the relaxation rate is independent of current and the ratio $j_{\text {exc }} / j_{c}$ is independent of temperature, in the other, with lower temperature, the relaxation rate is affected by the transport current, and the ratio $j_{\text {exc }} / j_{c}$ is a function of temperature.

\section{References}

Bezugly E V, Bratus E N and Galaiko V P 1977 Fiz. Nizk. Temp. 31010

Churilov G E, Svetlov V N and Dmitriev V M 1986 Fiz. Nizk. Temp. 12425

Ivlev B I, Kopnin N B and Maslova L A 1980 Zh. Eksp. Teor. Fiz. 781963

Ivlev B I and Kopnin N B 1984 Ukr. Fiz. Zh. 142435

Skocpol W J, Beasley M R and Tinkham M 1974 J. Low Temp. Phys. 16145 